\title{
Nanowire based heterostructures: Fundamental properties and applications
}

\author{
Martin Heiss, Carlo Colombo and Anna Fontcuberta i Morral \\ Laboratoire des Materiaux Semiconducteurs, École Polytechnique Fédérale de Lausanne, \\ Station 12, 1015 Lausanne, Switzerland
}

\begin{abstract}
In this paper the fundamental properties of heterostructures based on semiconductor nanowires synthesized with molecular beam epitaxy are reviewed. Special focus is given on surface passivation mechanisms with radial epitaxial passivation shells. The growth of radial p-i-n junctions in GaAs nanowires is discussed. Characterization of such nanowires on a single nanowire level is presented. The fundamental limits of single nanowire optical device performance are obtained by numerical simulation and discussed.
\end{abstract}

Keywords: Semiconductor nanowires, Solar energy conversion, Nanoepitaxy

\section{INTRODUCTION}

Semiconductor nanowires are expected to play an important role in the technological innovations of this century. In particular, they have attracted significant attention because they can be potentially used are in the energy conversion domain such as third generation solar cells or water splitting devices. Third generation solar cells aim to exploit novel physical phenomena in order to provide alternatives to traditional bulk and thin film devices. The main objective is to improve the ratio between conversion efficiency and total cost. ${ }^{1,2}$ Particular attention has been given to core-shell radial p-i-n structures, in which light absorption is orthogonal to the carrier collection. It is now widely accepted that such structures enable an extremely efficient carrier collection, as the minority carrier diffusion length can in all cases be shorter than the optical absorption length. ${ }^{3}$ This relaxes the demand for the material quality, which at the same time can lead to the reduction in material related costs. Supplementary advantages in using nanowires for photovoltaic applications are the improvement in the light collection trough index matching or enhanced light scattering and the minimization of the amount of material used. ${ }^{4}$ Moreover, it is known that lattice mismatched materials can be integrated when grown in the form of nanowires. This enables both the formation of strain-free multi-junction solar cells and the utilization of a cheap substrate as a support or part of the cell. ${ }^{5-7}$ Molecular beam epitaxy (MBE) grown GaAs nanowires are especially suitable for the fabrication of nanowire solar cells. From one side, MBE is a versatile technique. Axial and radial growth can be switched at convenience. Layers grown on the radial direction can be obtained with atomic precision and perfect epitaxial relation. ${ }^{8}$ The quality of the obtained interface is MBE-grade, which is an essential point for the fabrication of an efficient solar cell. Additionally, GaAs nanowires can be obtained without the use of gold, which is generally an unwanted impurity in the semiconductor industry. ${ }^{9,10}$ Finally, GaAs is one of the direct band gap semiconductors whose absorption is best matched with the solar spectrum. ${ }^{11}$

\section{RADIAL EPITAXIAL GROWTH ON THE SIDE FACETS OF NANOWIRES AND SURFACE PASSIVATION}

The nanowires reviewed here were obtained by the gallium-assisted catalyst-free method, ${ }^{12}$ where a gallium droplet is used for initiating and fostering the one-dimensional growth of GaAs. The growth mode of the nanowire can be changed from the axial to a radial growth by increasing the $\mathrm{As}_{4}$ beam flux to $5 \cdot 10^{-5} \mathrm{mbar}$ and lowering the temperature to $465^{\circ} \mathrm{C} .{ }^{8,13}$ The increased As flux will lead to a consumption of the Ga catalyst droplet and stop the axial growth. The Ga flux can be also increased to $2 \AA / \mathrm{s}$ thus increasing the radial growth rate. We have demonstrated that these conditions are ideal for growth on $\{110\}$ nanofacets. ${ }^{13}$ It is furthermore

\footnotetext{
E-mail: martin.heiss@epfl.ch
}

Nanoepitaxy: Materials and Devices III, edited by Nobuhiko P. Kobayashi, A. Alec Talin, M. Saif Islam, Proc. of SPIE Vol. 8106, 810603 - (c) 2011 SPIE - CCC code: 0277-786X/11/\$18 · doi: 10.1117/12.896471 
well known from literature that the relative low growth temperature and high arsenic fluxes are necessary in order to obtain a good decomposition of the incoming $\mathrm{As}_{4}$ flux on the non-polar $\{110\}$ surfaces and achieve defectfree epitaxial planar growth. During the radial growth by modulating additional incoming fluxes, specifically aluminium, it becomes possible to grow prismatic heterostructures in the Al-Ga-As material system. ${ }^{8}$ Recently we have furthermore demonstrated the growth of InAs quantum dots decorating the facets of nanowires. ${ }^{14}$

In addition to enabling the growth of low dimensional quantum systems, radial heterostructures with larger band gap materials further allow to efficiently passivate the nanowire core. To understand this we have studied the effect of diameter and surface passivation on the photoluminescence of zinc-blende GaAs nanowires. ${ }^{15}$ For this nanowires were grown under conditions where they present a slight tapering. This enables the measurement of diameter dependent effects by performing the characterization at different positions along the nanowire with different thickness. In order to investigate the effect of the nanowire surface on the optical properties the photoluminescence of an unpassivated nanowire sample is compared with a sample passivated by a prismatic epitaxial AlGaAs/GaAs shell.

\subsection{Experimental}

The nanowires used in the study of this section were grown using the gallium-assisted method in a GEN II Molecular Beam Epitaxy system. ${ }^{9}$ Nanowires composed of predominantly zinc-blende structure are obtained on $\mathrm{SiO}_{2}$ coated GaAs (111) substrates. During the longitudinal stage of growth the temperature was $630{ }^{\circ} \mathrm{C}$, the $\mathrm{As}_{4}$ beam flux $3 \cdot 10^{-6}$ mbar and the Ga rate equivalent to a planar growth of $0.22 \AA \mathrm{s}^{-1}$. Nanowire samples with and without a prismatic epitaxial shell were grown to study the influence of passivation on the properties of the samples. For both samples the longitudinal growth time was $10800 \mathrm{~s}$. This corresponds to a total nanowire length of approximately $15 \mu \mathrm{m} .{ }^{12}$

The second sample was passivated with a prismatic epitaxial capping. ${ }^{8}$ For this the nanowires were overgrown by an epitaxial shell of AlGaAs/GaAs at an $\mathrm{Al}$ rate of $0.5 \AA \mathrm{s}^{-1}$ and a Ga rate of $1.0 \AA \mathrm{s}^{-1}$. In order to achieve conditions suitable for planar growth on (110) facets, the $\mathrm{As}_{4}$ pressure in the chamber during radial growth was increased to $3.4 \cdot 10^{-5}$ mbar. ${ }^{8,13}$ This growth corresponded to a nominal planar deposition of $200 \AA$ $\mathrm{Al}_{0.33} \mathrm{Ga}_{0.66}$ As and $200 \AA$ GaAs. According to a calibration performed by Heigoldt et al., the growth on the lateral facets of the nanowires standing perpendicular to the substrate is approximately $20 \%$ of the nominal planar deposition. ${ }^{13}$ This is equal to an actual prismatic growth on the nanowire sides of $4 \mathrm{~nm}$ AlGaAs and $4 \mathrm{~nm}$ GaAs.

\subsection{Optical properties of unpassivated nanowires}

In this section the effects of the nanowire diameter for both capped and bare nanowires are studied. The nanowires are dispersed on $\mathrm{Si} / \mathrm{SiO}_{2}$ substrates patterned with marker symbols that allow to locate a specific nanowire in subsequent measurements. First the optical properties are characterized with spatially resolved micro-photoluminescence spectroscopy. Subsequently the nanowire diameter is obtained by Atomic Force Microscopy. By correlating both datasets, the diameter dependence of the optical properties can be studied for both bare and passivated nanowires. Quantitative comparison of the two samples allows to study the effect of the surface on the optical properties.

Due to the abrupt discontinuity of the crystal lattice at semiconductor surfaces, a large number of localized energy states can be introduced to the surface region. These energy states can significantly increase the recombination rate in the surface region. ${ }^{16}$ Furthermore oxidation of the surface and other inhomogeneities on the crystal facets generally can lead to non-radiative recombination and a degradation of the photoluminescence signal. ${ }^{17,18}$ An optically inactive layer at the semiconductor surface can be the consequence. ${ }^{19}$ Such phenomena will be most pronounced for thin nanowires where the surface to volume ratio increases.

In the following the diameter dependence of the photoluminescence will be compared for nanowires with and without a passivation layer. Controlling the influence of the surface on the optical and electronic properties is a very essential prerequisite for future more complex nanowire based devices. We can demonstrate that capping of the nanowires with an epitaxial AlGaAs layer can significantly improve the optical properties of the nanowire. Due to the larger band gap of AlGaAs than GaAs, a barrier to the nanowire surface can be obtained that confines the carriers to the core of the nanowire, thus significantly enhancing the optical properties. 

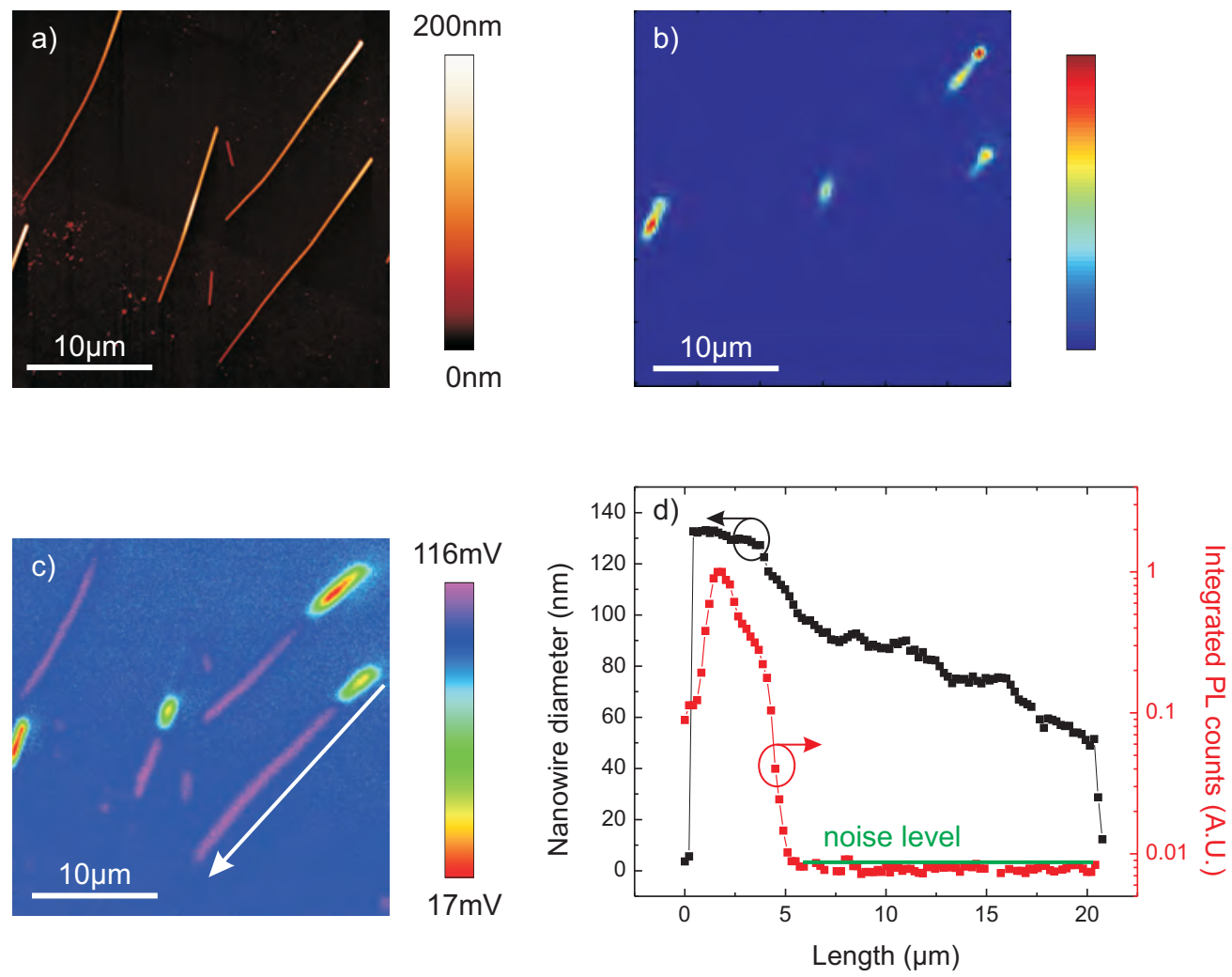

Figure 1. a) Atomic force micrography of uncapped nanowires transferred to a $\mathrm{SiO}_{2}$ substrate. b) Mapping of integrated $4.2 \mathrm{~K}$ micro-photoluminescence signal. c) Confocal reflectivity mapping of the sample surface. The measurements a)-c) are all performed on the same area of the sample. d) Dependence of nanowire diameter and integrated photoluminescence along the nanowire length as indicated by the arrow in c).

Figure 1a) shows Atomic Force Microscopy imaging of the unpassivated GaAs nanowire sample transferred to a $\mathrm{SiO}_{2}$ substrate. Spatial mapping of the integrated micro-photoluminescence signal obtained from the same sample area at $4.2 \mathrm{~K}$ is plotted in Figure 1b), while mapping of the reflected laser light is shown in Figure 1c). The excitation in the wire was generated using a semiconductor laser diode $(780 \pm 10 \mathrm{~nm})$ focused to an diffraction limited laser spot on the surface, with an excitation power density of $250 \mathrm{~W} \mathrm{~cm}^{-2}$. Circularly polarized incident light was used to ensure that all linear polarized states in the nanowire are excited with equal probability. The thick parts of the nanowires in Figure 1 show a strong integrated photoluminescence signal. Concurrently the reflected laser light is strongly reduced at these spots.

This behavior is illustrated in detail in Figure 1d), which shows the thickness of the nanowire along the axis. The nanowire exhibits a negative tapering in the order of $0.5 \%$ along the length. Due to the small tapering, the nanowire diameter can be approximated to be homogeneous over the micro-photoluminescence laser excitation spot size. Figure 1d) furthermore shows the integrated photoluminescence intensity along the nanowire axis. The maximum of photoluminescence intensity is observed in the approximately $140 \mathrm{~nm}$ thick part of the nanowire. With the nanowire radius decreasing, the photoluminescence signal rapidly vanishes. It approaches the detection limit of the spectrometer system for a integration time of $1 \mathrm{~s}$ at nanowire diameters below approximately $100 \mathrm{~nm}$.

When correlating the measurements of the nanowire photoluminescence intensity and diameter obtained by atomic force microscopy one has to consider that the the resolution of the photoluminescence measurements is significantly lower compared to the lateral resolution of the atomic force microscopy data. Therefore, the correlation between the two measurements in Figure 1d) was performed under the assumption that the position of the wire with the largest photoluminescence intensity corresponds to the part of the nanowire with the largest diameter. This allows to directly plot the photoluminescence signal with respect to diameter.

Literature values of the absorption length of GaAs for the used excitation laser energy $(1.59 \mathrm{eV})$ are in the 
a)

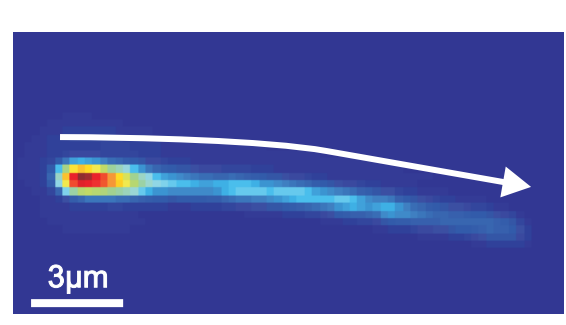

b)

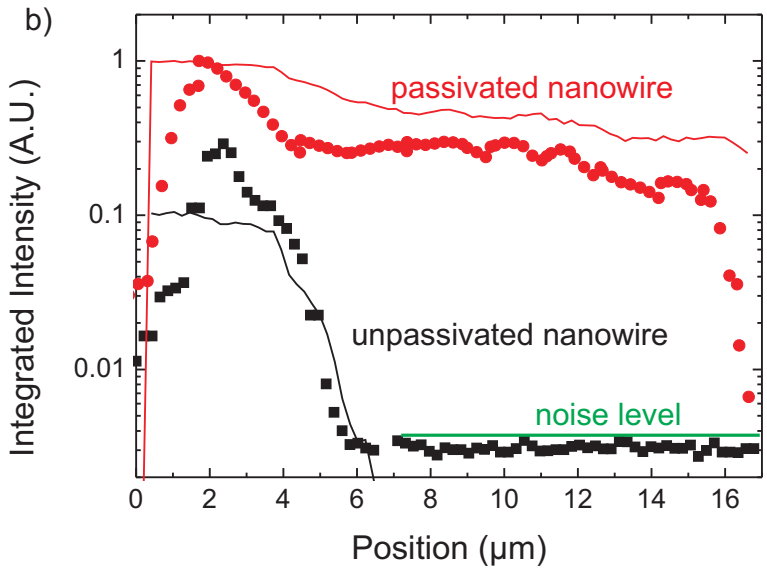

Figure 2. a) Mapping of integrated photoluminescence signal for a AlGaAs/GaAs epitaxially overgrown nanowire otherwise grown at the same conditions of the sample in Figure 1. b) Integrated photoluminescence signal along the nanowire length indicated by the white arrow in a) (red circles); For comparison the integrated intensity for the uncapped nanowire in Figure 1d) is drawn with the intensity in scale (black squares). For reference also the normalized nanowire cross section $\pi(d / 2)^{2}$ (red line) and nanowire cross section reduced by $45 \mathrm{~nm}\left(\pi(d / 2-45 \mathrm{~nm})^{2}\right)$ drawn (black curve).

order of $1 \mu \mathrm{m},{ }^{20}$ much larger compared to the typical nanowire diameters in the sample. Absorption in nanowires should therefore occur approximately homogeneously throughout the nanowire. In the absence of any surface related nonradiative recombination mechanisms the absorption and photoluminescence intensity of the wires would only be related to the volume of semiconductor material and therefore proportional to the cross section area $\left(\pi(d / 2)^{2}\right)$ of the wire. However for unpassivated nanowires the integrated photoluminescence intensity is not proportional to the cross section volume $\pi(d / 2)^{2}$. Instead, it drops rapidly to the noise level for diameters below approximately $100 \mathrm{~nm}$. No significant photoluminescence could be observed from this sample for diameters below this value. This is a obvious sign that in unpassivated nanowires the optical activity is not homogeneous along the nanowire. The rapid decrease with diameter could indicate that an optically inactive layer exists on the nanowire surface.

\subsection{Influence of the passivation layer}

In the following we will show that a lateral capping of the nanowires with an epitaxial shell can significantly reduce the strong diameter dependence described above. Figure 2 shows a nanowire from the sample also grown with an $\mathrm{As}_{4}$ flux of $3 \cdot 10^{-6}$ mbar, but subsequently passivated with an epitaxial AlGaAs/GaAs shell. The morphology of the passivated nanowires is otherwise similar to the unpassivated sample. Figure 2a) shows the integrated photoluminescence intensity that exhibits a detectable signal along the entire length of the nanowire. Figure 2b) presents the integrated photoluminescence intensity along the nanowire as indicated by the white arrow. For a direct comparison, the integrated signal of the unpassivated nanowire sample is drawn in the graph. Care was taken to ensure that the integrated intensities can be compared with the measurements in Figure 1 . The passivated nanowires show a four-fold signal enhancement in the thick $(\approx 140 \mathrm{~nm}$ diameter $)$ part of the wire with respect to the equally thick parts of the unpassivated nanowires. In the region of the nanowire with diameters below $100 \mathrm{~nm}$ the passivation results in an increase of photoluminescence signal by more than two orders of magnitude. This shows that the epitaxial passivation significantly diminishes surface effects and thereby can improve the optical properties by several orders of magnitude.

Moreover, the comparison of the photoluminescence from the passivated and unpassivated nanowire samples with two different geometrical factors delivers interesting results. First the normalized cross section area $\pi(d / 2)^{2}$ of a typical nanowire from the sample is drawn in Figure 2. Furthermore, a reduced cross section curve $(\pi(d / 2-$ $45 \mathrm{~nm})^{2}$ ) is drawn, corresponding to a nanowire cross-section where the effective radius is reduced by $45 \mathrm{~nm}$. The photoluminescence signal from the passivated nanowire is proportional to the full nanowire cross section area. This indicates that the whole nanowire is optically active and does not suffer from pronounced surface effects. In contrast, the integrated photoluminescence signal from the unpassivated nanowire is proportional to the second 
geometrical factor that assumes a $45 \mathrm{~nm}$ reduced radius. This is a clear indication that the outermost $45 \pm 5 \mathrm{~nm}$ of the unpassivated nanowires are optically inactive. A typical reason for such an optically inactive zone could be the formation of a space charge region at the surface. ${ }^{19}$ The different surface recombination mechanisms that could account for the observed behavior are discussed in the following section.

\subsection{Surface recombination mechanisms in nanowires}

Several factors may affect the photoluminescence intensity in the presence of a surface. Two major near-surface effects affecting photoluminescence intensity in direct band gap semiconductor materials are reported in literature: surface recombination ${ }^{21,22}$ and surface band bending. ${ }^{19,23}$

Most literature studies investigated surface recombination mechanisms in bulk material. However, for the radial nanowire geometry the surface to volume ratio can induce changes on the surface recombination mechanisms. The sample studied in Section 2.2 showed a pronounced diameter dependence of emitted photoluminescence light. In the following the diameter dependence of these effects will be deducted from theoretical considerations. Due to the radial geometry of the nanowires, the surface to volume ratio increases with decreasing diameter. This could in general account for the experimentally observed diameter dependence of photoluminescence.

\subsubsection{Surface recombination}

The abrupt discontinuity of the crystal lattice at a semiconductor surface may introduce a large number of localized energy states at the surface region. ${ }^{16}$ The total number of carriers recombining at the surface per unit area $\left(R_{s}\right)$ can be described by the Shockley-Read-Hall statistics. ${ }^{22}$ For typical experimental conditions a surface recombination velocity $\mathrm{S}$ can be defined that fulfills the relation $R_{s}=S n$, where $\mathrm{n}$ is the excess minority carrier density. ${ }^{21}$ When assuming flat band conditions at the surface, the recombination process of a planar surface can be described by the diffusion equation: ${ }^{21}$

$$
D \nabla^{2} n-\frac{n}{\tau}+g e^{-\alpha x}=0
$$

where $D$ is the carrier diffusion coefficient, $\tau$ the total carrier lifetime, $g$ the generation rate of carriers by the optical excitation, and $\alpha$ the optical absorption coefficient.

The absorption length of GaAs for the excitation laser energy of $1.59 \mathrm{eV}$ used in our experiments is in the order of $1 \mu \mathrm{m},{ }^{20}$ much larger than the typical nanowire diameters in the sample. For the nanowire geometry, the generation $g$ of carriers can be considered constant throughout the cross section area, and the exponential in Equation 1 can be neglected. We will furthermore consider a radial symmetric nanowire. Therefore, the diffusion equation for the system can be solved in cylinder coordinates. Due to the large aspect ratio, the nanowire can be considered quasi infinite along the z-axis. Therefore a gradient of the carrier concentration not parallel to the nanowire radius can be neglected. In this way Equation 1 can be transformed to:

$$
\frac{D}{r} \frac{\partial}{\partial r} r \frac{\partial n(r)}{\partial r}-\frac{n(r)}{\tau}+g=0
$$

In the following, Equation 2 will be considered in the limit of long overall carrier lifetimes $\tau$. Namely, the carrier diffusion length $L=\sqrt{D \tau}$ should be much larger than the nanowire radius. In this limit it is possible to obtain a simple analytical solution of Equation 2 with the boundary conditions $\left.\frac{\partial n(r)}{\partial r}\right|_{r=r_{0}}=\frac{S}{D} n\left(r_{0}\right)$ and $\left.\frac{\partial n(r)}{\partial r}\right|_{r=0}=0$ :

$$
n(r)=\frac{-g r^{2}}{4 D}+\frac{g r_{0}^{2} S+2 g r_{0} D}{4 D S}
$$

Here $r_{0}$ refers to the overall nanowire radius. The photoluminescence emitted from the structure can then be estimated to be proportional to the excess carrier density $n(r) .{ }^{21}$ By integrating $n(r)$ over the nanowire cross section and normalizing to the cross section area one obtains the following equation: 
a)

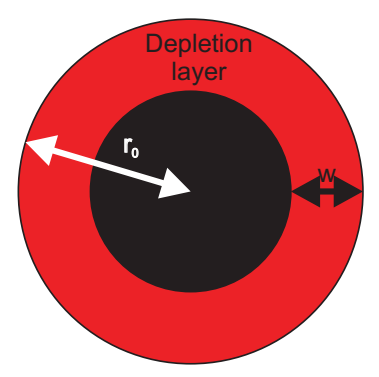

b)

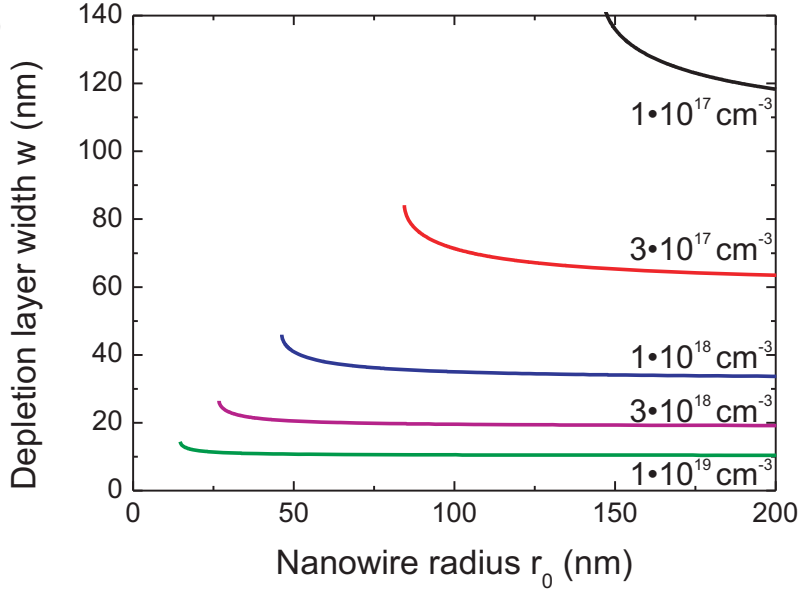

Figure 3. a) Schematic of a radial symmetric nanowire with radius $r_{0}$ and depletion layer of width w. b) Calculated depletion layer width in dependence of the nanowire radius.

$$
I \propto \frac{1}{\pi r_{0}^{2}} \int_{0}^{r_{0}} 2 \pi r \frac{n(r)}{\tau_{\text {rad }}} d r,
$$

where $I$ is proportional to the average luminescence per unit volume of the nanowire, and $\tau_{\text {rad }}$ ist the radiative recombination lifetime. Again for simplicity reabsorption processes will be neglected due to the typically long absorption lengths compared to the nanowire diameter. By substitution of Equation 3 in 4 and solving the integral, one obtains:

$$
I \propto \frac{g}{2 S} r_{0}+\frac{g}{8 D} r_{0}^{2} .
$$

Equation 5 describes the dependence of average luminescence per unit volume on the radius $r_{0}$ of the nanowire. The value is already normalized to the purely geometrical factor arising from the change in nanowire volume. In general, the dependence of photoluminescence intensity on the nanowire radius $r_{0}$ is found to exhibit a mixture of linear and quadratic terms of $r_{0}$. It is important to keep in mind that Equation 5 is only valid for nanowire diameters much smaller than the absorption length of light in the semiconductor.

Depending on the relative ratio of the coefficients $\mathrm{S}$ and $\mathrm{D}$, two extreme regimes can be distinguished. In the regime $r_{0} S \ll D$ (recombination-limited), a linear dependence on nanowire radius is expected. In contrast, a diffusion-limited regime can be expected in for the limit $r_{0} S \gg D$. In this limit, a quadratic dependence of the photoluminescence intensity per unit volume on the nanowire radius is predicted.

\subsubsection{Surface band bending}

In this section, the effect of a surface space charge region in unpassivated nanowires will be discussed. The nanowires investigated for the current study have side facets of the $\{110\}$ family. Ideally, these non-polar surfaces are known to present a low quantity of surface states and no band bending when exposed to vacuum. ${ }^{24,25}$ Unfortunately, the nanowires are exposed to ambient conditions after removal from the growth chamber. This causes the formation of a thin oxide layer on the nanowire surfaces. ${ }^{26}$ Such oxides are known to cause a change of the surface Fermi level also on $\{110\}$ surfaces. ${ }^{27,28}$ This can lead to a bending of the bands at the surface. ${ }^{24}$

In a semi classical model the band bending can be explained considering the difference $\Phi$ of the Fermi level at surface states with respect to the Fermi level of the bulk. In an idealized system the pinning occurs exactly at the center of the band gap. Landgren et al. have experimentally determined the surface Fermi level of of oxidized (110) GaAs. They obtained values of $0.5 \mathrm{eV}$ above the valence band for p-type material and $0.6 \mathrm{eV}$ below the conduction band for n-type GaAs respectively. ${ }^{28}$ 
The difference in Fermi level at the surface with respect to the inner material leads to the formation of a space charge region at the surface. The actual width of such a space charge region is mostly affected by the doping level. With increased doping the electric field is screened more efficiently, therefore reducing the depletion region. For a planar geometry, the width of the depletion region is given by:

$$
w=\sqrt{\frac{2 \epsilon \epsilon_{0} \Phi}{e N_{D}}}
$$

where $N_{D}, \epsilon, \epsilon_{0}$ and $e$ are the doping concentration, dielectric constant in the material, dielectric constant in the vacuum, and electron charge, respectively. ${ }^{29}$

The effective reduction in diameter by $40-50 \mathrm{~nm}$ observed in unpassivated nanowires could be explained in the frame of such a surface depletion model. Indeed the electric field present in the space charge region can lead to a separation of carriers that can significantly reduce the optical activity. ${ }^{19,23}$

In a nanowire, the electrostatics change due to the cylindrical geometry. For the following considerations, effects from the hexagonal structure will be neglected, and a rotation invariant nanowire with radius $r_{0}$ and homogeneous doping concentration $N_{D}$ will be considered. Therefore, the Poisson equation for the system can be solved in cylinder coordinates. Due to the large aspect ratio, the nanowire can be considered quasi infinite along the z-axis. Consequently, components of the electric field not parallel to the nanowire radius can be neglected. The Poisson equation within the space charge region in the nanowire is then given by:

$$
\frac{1}{r} \frac{\partial}{\partial r} r \frac{\partial \phi(r)}{\partial r}=-\frac{e N_{D}}{\epsilon \epsilon_{0}}
$$

Solving this equation using the appropriate boundary conditions of vanishing electric field in the nanowire and surface potential $\phi\left(r_{0}\right)=\Phi$ one obtains an implicit equation for the nanowire depletion layer width $w$ in dependence of the nanowire radius $r_{0}$ :

$$
-\frac{w^{2}}{2}+r_{0} w-\left(r_{0}-w\right)^{2} \ln \left(\frac{r_{0}}{r_{0}-w}\right)=\frac{2 \epsilon \epsilon_{0} \Phi}{e N_{D}} .
$$

From Equation 8 it is directly apparent that the depletion width in the nanowire in general also depends on the nanowire radius $r_{0}$. In the limit $r_{0} \rightarrow \infty$, Equation 8 approaches Equation 6 for the bulk case. For the limit of full depletion of the nanowire $\left(r_{0} \rightarrow w\right)$, the depletion layer width $w$ is increased by a factor of $\sqrt{2}$ with respect to the depletion width of the bulk case. The dependence of the depletion layer width on the nanowire radius is plotted in Figure 3b for different doping concentrations.

\subsection{Discussion}

Figure 4 summarizes the experimental diameter dependent data of unpassivated nanowires grown at an $\mathrm{As}_{4}$ flux of $3 \cdot 10^{-6}$ mbar as well as various theoretical approximations. All data are normalized to intensity per unit volume for a diameter of $200 \mathrm{~nm}$. The surface recombination model alone can not fully explain the full range of the experimental data. As discussed in Section 2.4.1, the diameter dependence of surface recombination should be a mixture of linear and quadratic terms and can only explain well the data between the curves of recombination-limited and diffusion-limited surface recombination regime (yellow area in Figure 4).

In Section 2.2 it was already discussed empirically that the signal level of photoluminescence in unpassivated nanowires drops very steeply in an approximately exponential way below the noise level for a radius below approximately $50 \mathrm{~nm}$. This behavior in principle could be explained with the presence of a surface band bending that causes an optically inactive zone near the surface. ${ }^{19,23}$ A plot of the surface band bending model discussed in the previous section is also shown in Figure 4. In the model, literature values for a typical potential barrier of oxidized GaAs $\Phi=0.5 \mathrm{eV}^{27,28}$ are used. As shown in Figure 4 such a surface depletion model can fit the experimental data very well. It is important to note that surface band bending also depends on the doping concentration. This indicates that a highly doped intentionally doped shell could offer another pathway to reduce the influence of surface band bending. 


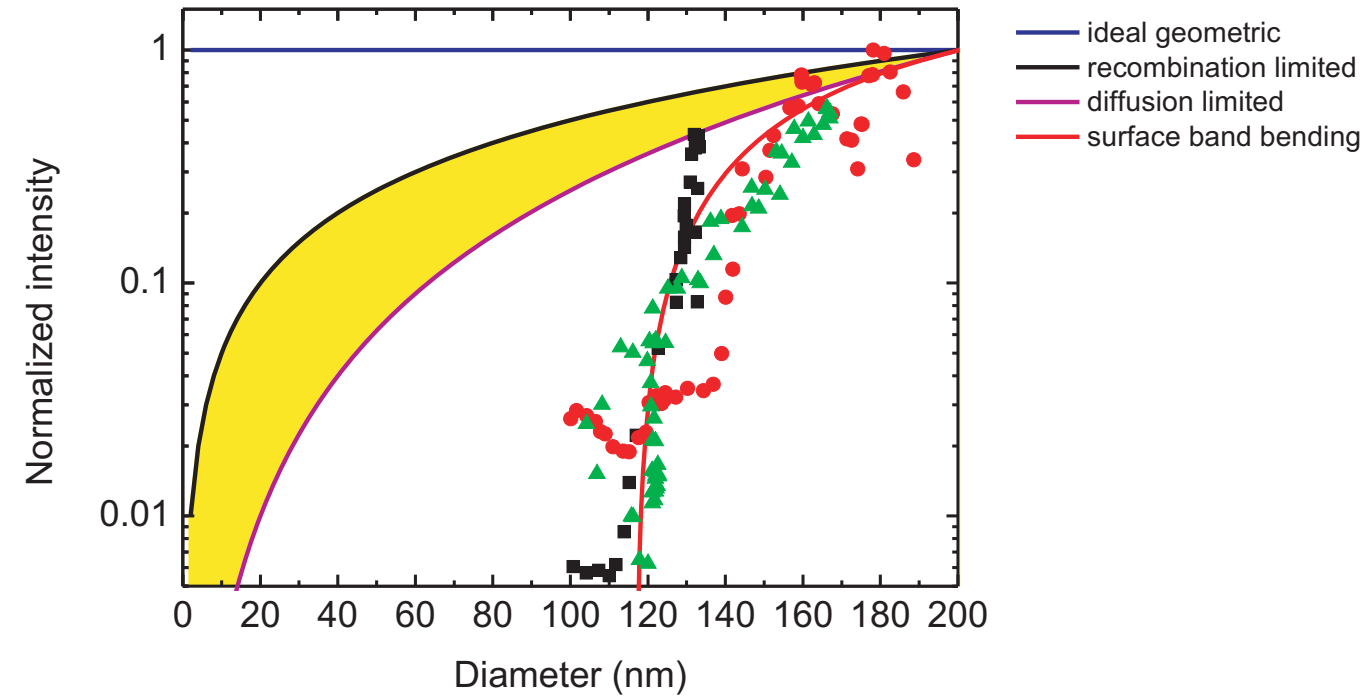

Figure 4. Comparison of the theoretical diameter dependences of photoluminescence intensity per unit volume. The geometrical effect $\left(\propto r_{0}^{2}\right)$ resulting from a reduction in nanowire volume is already separated off. All plots are normalized to the intensity at a nanowire diameter of $200 \mathrm{~nm}$. The curves correspond to the ideal geometric case in the absence of surface recombination mechanisms, surface recombination in the recombination limited regime, surface recombination in the diffusion limited regime, and surface band bending, respectively. The yellow-shaded area shows the possible range of surface recombination effects for a the general case with a mixture of linear and quadratic diameter-dependent terms.

It is important to keep in mind that the experimental data was acquired at low excitation powers in the order of $250 \mathrm{~W} \mathrm{~cm}^{-2}$. In literature, the presence of surface band bending under low excitation power conditions is commonly reported. ${ }^{22}$ The surface band bending in principle can be reduced under higher power illumination. When the optical excited carrier concentration is much larger than the equilibrium carrier concentration, flat band conditions can be achieved. Such conditions are typically difficult to achieve in steady-state photoluminescence, but can be obtained by high-power pulsed laser excitation of the sample. ${ }^{22}$ We have recently determined a value of the interface recombination rate in passivated GaAs nanowires of only $2.9 \cdot 10^{3} \mathrm{~cm} \mathrm{~s}^{-1}$ by investigating the minority carrier lifetime in dependence on nanowire diameter. ${ }^{15}$

\section{DOPING IN GAAS NANOWIRES}

Silicon is an amphoteric impurity in GaAs. This means that the incorporation of silicon can lead to n- or ptype doping, depending on whether it is incorporated in As or Ga sites. ${ }^{30}$ In the case of gallium-assisted GaAs nanowire growth, we have observed that the incorporation of $\mathrm{Si}$ in the growth results in a p-type doping.

For this growth conditions, due to the lower temperature and higher $\mathrm{As}_{4}$ beam flux, the incorporation of $\mathrm{Si}$ leads to a n-type layer. ${ }^{31}$ A detailed study on the doping mechanisms in catalyst free GaAs nanowires can be found elsewhere. ${ }^{32}$

\section{APPLICATION FOR P-I-N JUNCTION SOLAR CELLS}

We recently reported the growth of GaAs p-i-n nanowire structures synthesized in a Gen II MBE system. ${ }^{33}$ Two-inch (111)B GaAs wafers coated with a sputtered $10 \mathrm{~nm}$ thick silicon dioxide were used as substrates. The nanowire growth was carried out at a nominal GaAs growth rate of $0.25 \AA / \mathrm{s}, \mathrm{As}_{4}$ partial pressure of $2 \cdot 10^{-6} \mathrm{mbar}$, Ga rich conditions, a temperature of $630^{\circ} \mathrm{C}$ and with $7 \mathrm{rpm}$ rotation. As we have discussed in the previous section, the nanowires grown on coated (111)B GaAs wafers grow perpendicular to the substrate surface. The core of the p-i-n nanowire junction was doped type p. This was achieved by adding a silicon flux during the nanowire growth. For that, the silicon cell was heated with $13 \mathrm{~A}$, which corresponds to a nominal doping flux of $2 \cdot 10^{10} \mathrm{~cm}^{-2} \mathrm{~s}^{-1}$. 
a)

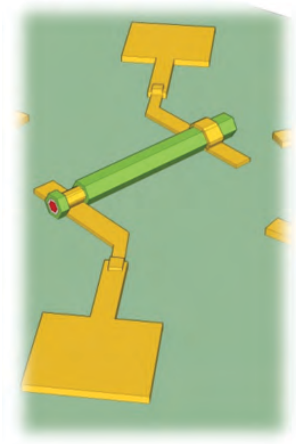

b)

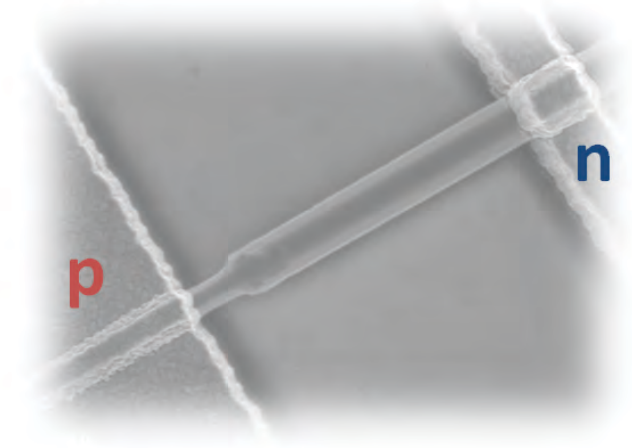

Figure 5. a) Sketch of the device structure b) SEM of a typical device. ${ }^{33}$

After $3 \mathrm{~h}$ of growth, the nanowires presented a total length of $9 \mu \mathrm{m}$. Then, the growth was stopped and the conditions were changed toward planar MBE growth. The As 4 partial pressure was increased to $5 \cdot 10^{-5}$, which resulted in the crystallization of the Ga droplet. The fabrication of the p-i-n junction continued by the epitaxy of a $15 \mathrm{~nm}$ intrinsic and a $50 \mathrm{~nm}$ n-type layer on the facets of the nanowires. The n-type doping was obtained by adding silicon during growth.

In order to study the fundamental properties of such semiconductor nanowires it can be beneficial to consider the performance of a single nanowire device. For this the p-i-n nanowire structures can be transferred on oxidized silicon substrate and contacted with standard lithography techniques. The p-type core of the nanowire has to be contacted separately. This is achieved by first etching a section of the n-type and intrinsic layers of the nanowire. The etching can be performed with a citric acid solution with a calibrated etch rate. We typically use $\mathrm{Pd} / \mathrm{Ti} / \mathrm{Au}$ to form an Ohmic contact with the p-type core. The n-type shell can be directly contacted in a second lithographic step and evaporating a $\mathrm{Ti} / \mathrm{Au}$ layer. Details of the contacting procedure can be found in. ${ }^{33}$

A sketch of the contacted p-i-n nanowire structure is presented in Figure 5a. There the coaxial layers can be observed, as well as the contacting of the inner and exterior layer. A scanning electron micrograph of a real structure that we have realized is shown in Figure 5b. The microscopic contacts can be used for the measurement of the electrical response in the dark and under illumination. We have measured the current-voltage characteristic of the contacted p-i-n nanowire in the dark and under various illumination conditions including 1.5 AM. ${ }^{33}$

Typical I-V characteristics in the dark and under illumination are shown in Figure 6a. A typical diode behavior is obtained. A photo current is present upon illumination of the device. In order to characterize the device current-voltage characteristics were first measured as a function of the illumination intensity at room temperature. ${ }^{33}$ The nanowire was illuminated with a diode laser with excitation wavelength of $780 \pm 10 \mathrm{~nm}$. In order to cover the whole nanowire the incident beam was focused to a spot of approximately $15 \mu \mathrm{m}$. The relation between the generated photo current and the excitation is shown in Figure 6. From 1 to $189 \mu W$, we observed a linear relation in log-log scale between the photo current and the excitation power.

In order to understand the origin of the photo current we have realized spatially resolved measurements. For this the contacted nanowires were mounted on an $\mathrm{x}-\mathrm{y}$ piezo stage of a confocal microscope. The confocal lens of the microscope was used to illuminate the nanowire with a spot of about $1 \mu m$ in diameter. This allowed to perform scanning photo current microscopy of the device. The short circuit current was mapped scanning the area around the nanowire in grid every $250 \mathrm{~nm}$. The image obtained superimposed to the SEM picture of the same area is shown in Figure 7a. When the illumination spot is located on the uncovered p-type region, no photo current is observed. An increase in the photo current is only observed when the p-i-n junction is illuminated. ${ }^{33}$ The response is found to be homogeneous along the wire. This indicates the existence of a homogeneous deposition of the intrinsic and n-type layers on the nanowire facets. 
a)

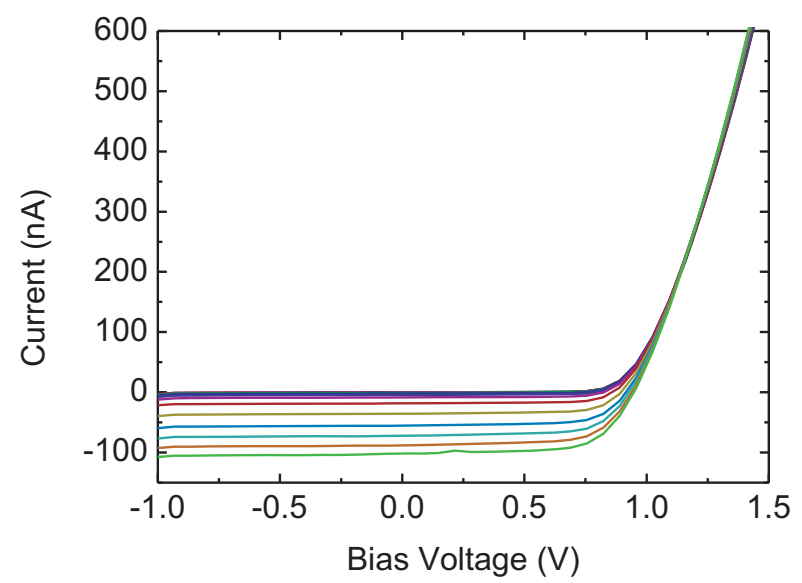

b)

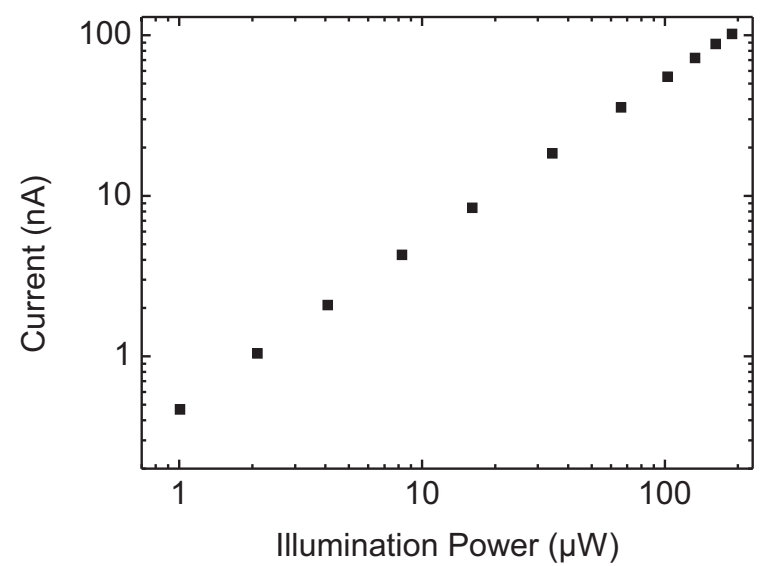

Figure 6. a) Nanowire p-i-n junction current-voltage measurements in the dark and under various illumination powers b) Short-circuit current as a function of the illumination power, corresponding to the measurements in a) ${ }^{33}$

For further characterization of the p-i-n junction, the light emitted by the nanowire in forward bias was measured. This corresponds to the regime in which the junction works as a light emitting diode. A typical spectrum is shown in Figure 7b. The emission peak at $1.42 \mathrm{eV}$ is in good agreement with the room temperature band gap of GaAs. The existence of electro luminescence at room temperature constitutes a further proof of the quality of the nanowires and the p-i-n junction. A cooling of the sample to $4.2 \mathrm{~K}$ further increased the optical activity. Figure $7 \mathrm{c}$ shows a optical microscopy imaging of the cooled sample under forward bias. The light emitted from the radial nanowire p-i-n junction can be clearly observed with the CCD camera of the confocal microscope, and is consistent with the photo current mapping in Figure 7a.

In order to get the efficiency of the nanowire device it was also measured under 1.5 AM illumination conditions. To get a parameter such as power conversion efficiency it is necessary to divide the maximum generated power density by the total incident energy density at $1.5 \mathrm{AM} .{ }^{33}$ As indicated by the scanning photo current measurements a first estimation for the effective device area to be considered is the projected area of the p-i-n junction. This area can be obtained from scanning electron micrography and corresponds to the product of length L and diameter of the nanowire in the p-i-n region. For the best sample a value of $4.5 \%$ was obtained. ${ }^{33}$ The fill factor was 0.65 .

a)

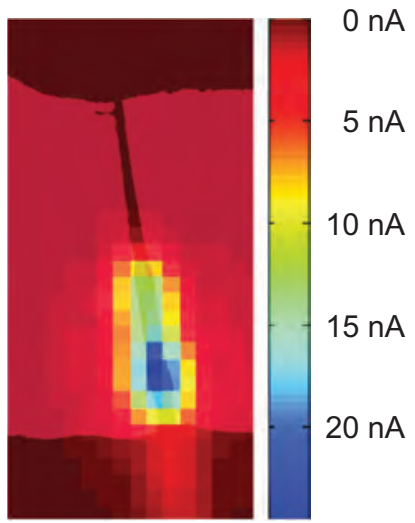

b)

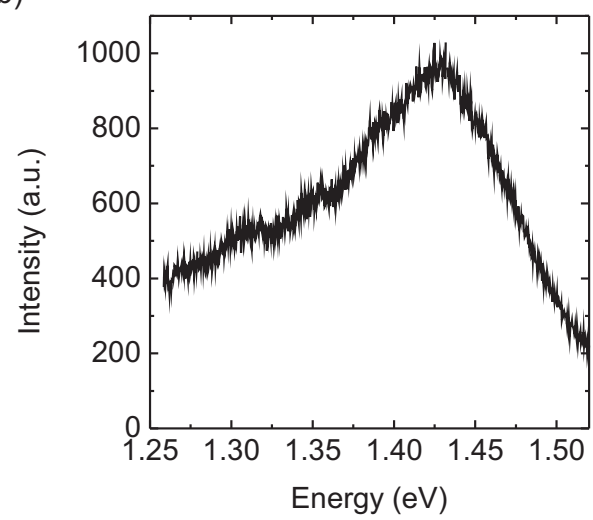

c)

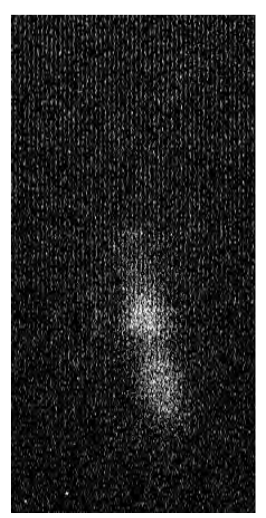

Figure 7. a) Scanning photo current map of the nanowire p-i-n device b) Light spectrum of the of the p-i-n junction emitting in forward bias light emitting diode mode. ${ }^{33}$ c) Microscopic image of the sample in forward bias ( $\left.\mathrm{I}=2.6 \mu \mathrm{A}\right)$ light emitting mode. For this measurement the sample was cooled to $4.2 \mathrm{~K}$. 
a)

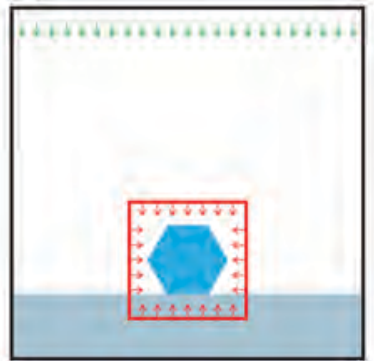

b)

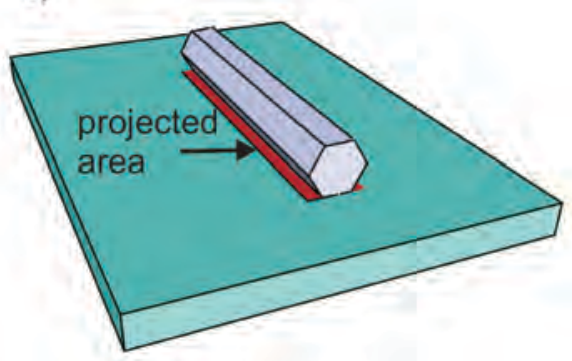

Figure 8. a) Sketch of the 2D-simulation geometry. We calculate the net flux of energy through a box around the nanowire (red) and normalize it to the flux through the projected area of the nanowire on the substrate as obtained by a reference simulation. b) Experimental condition considered in simulation. The hexagonal nanowire is placed on a planar substrate and illuminated with a plane wave light perpendicular to the substrate surface. See also Figure 5.

\section{LIMITING EFFECTS IN SINGLE NANOWIRE SOLAR CELLS}

To date several groups have carried out similar approach for nanowire solar cells ${ }^{34-37}$ with device characterization based on a single nanowire level that is placed on a substrate and illuminated perpendicular to the nanowire axis. However, while this geometry is technically less challenging compared to a single nanowire device it imposes certain fundamental limits on the device performance. First of all the effective material thickness that is present for the light absorption is significantly smaller compared to a illumination parallel to the axis. Especially for photon energies close to the band gap, where the absorption length is typically much longer than the nanowire diameter this might significantly reduce the light absorption. On the other hand it is well known that optical resonances in the visible light spectral range can exist for nanowires in the orders of hundred nanometers. This can increase the light absorption beyond the purely geometrical limit for certain wavelength. In general these counteracting phenomena can lead to a significant deviation of the actually absorbed photons with respect to the geometrically incident photons. Certainly this can lead to an over or underestimation when deducting the power conversion efficiency based on the geometrical area of the junction. To investigate the magnitude of this effect we have performed finite difference time domain simulations, also taking into account the effect of the substrate and the nanowires.

For the simulations the hexagonal GaAs nanowire is placed on a semi infinite substrate with either $\epsilon=1$ (no substrate), $\epsilon=2.25$ (glass) or $\epsilon=-\infty$ (a perfectly reflecting electrical conductor). The illumination is performed using a plane wave incident from the top of the simulation area that is polarized either parallel or perpendicular to the nanowire axis. The calculations are performed using literature values for the wavelength dependent real and imaginary parts of the dielectric functions of GaAs. ${ }^{38}$ We use perfectly matched layers as boundary conditions. One should note that we only consider the absorption in intrinsic material and neglect changes in the absorption coefficient induced by doping. The nanowire absorption is obtained by calculating the net flux inside a box around the nanowire and normalized to the flux through the geometrically projected area of the nanowire on the substrate, as obtained from a reference simulation without nanowire and substrate. In this way we obtain the effective fraction of the flux incident on the nanowire. We perform separate simulations for light polarized parallel and perpendicular to the nanowire axis.

The internal quantum efficiency (IQE) of a device corresponds to the efficiency of light conversion once the light has been absorbed in the device. The external quantum efficiency (EQE) refers to the one measured with respect to the incident photons. The ratio of EQE/IQE therefore corresponds to the ratio of actually absorbed photons with respect to the geometrically incident flux. We obtain this ratio directly from our simulations. Other calculations and experimental results indicate that the ratio EQE/IQE can be nearly 1 for nanowire or nanocone arrays. ${ }^{39-41}$ However this is far to be the case for single nanowires. Several factors affect the absorption in a nanowire positioned on a substrate (Figure 8b). It has been shown that light absorption can be significantly affected by the incident polarization and Mie scattering. ${ }^{42,43}$ As a consequence, the cross-section of absorption can significantly deviate from the geometrically projected area of the nanowire on the substrate. This can cause substantial deviations between the measured EQE and the actual IQE. When trying to optimize the device 


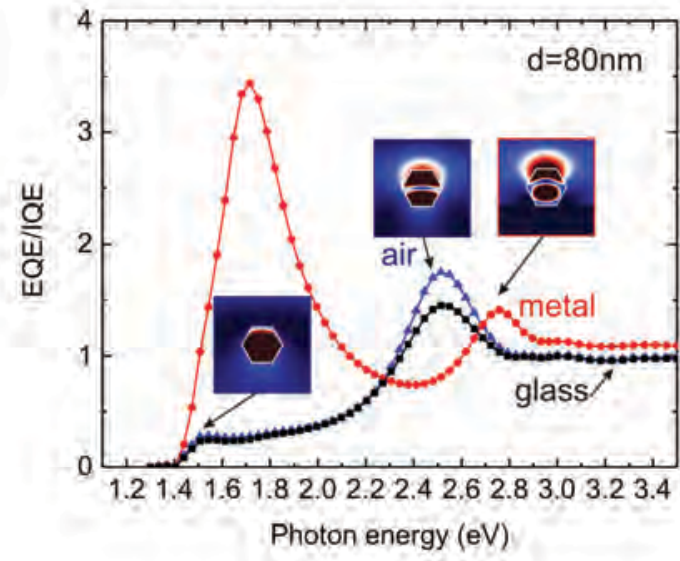

Figure 9. Ratio of external quantum efficiency to internal quantum efficiency (EQE/IQE) in dependence of photon energy for cases of nanowire in air $(\boldsymbol{\Delta})$, on glass substrate $(\boldsymbol{\square})$ and on an ideally reflecting metal surface $(\bullet)$. The data shown corresponds to incident light polarized parallel to the nanowire axis and a wire diameter of 80 nm. The inset shows the calculated electric field energy distribution for selected data points indicated by the arrows.

by single nanowire measurements this becomes of extreme importance. Also, it shows that the comparison of the efficiency of single nanowire devices of different diameters and/or positioned on different substrates is not straightforward. There has been a certain debate in literature on the magnitude of such deviations, it has been difficult to quantify and separate from other influences. ${ }^{44}$

The spectral and substrate dependence of a single nanowire with $80 \mathrm{~nm}$ diameter is shown in figure 9 . In a part of the spectral range the ratio can be considerably larger than one. This corresponds to the situation of Mie resonance where the light absorption can exceed the pure geometric fraction of the incident plane wave by a factor of more than 3. Meanwhile in other parts of the spectra the EQE/IQE ratio is smaller than one. This can be attributed to the spectral range where the absorption length is much larger than the actual nanowire diameter, thus quenching the absorption. The position of the resonances does not only depend on the considered nanowire diameter but also changes considerably with respect to the substrate used. The calculated spatial electromagnetic field energy distribution $\left(\sim E^{*} \cdot D+H^{*} \cdot B\right)$ obtained under resonant conditions is plotted in the inset of Figure 9. Interestingly the glass substrate dampens the Mie resonance in the nanowire and does not affect the position. This is quite different in the case of a perfectly reflecting metal substrate $(\epsilon=-\infty)$ where the simulations show a blue shift of the resonances. As GaAs is a direct band-gap semiconductor, the EQE/IQE ratio in a $80 \mathrm{~nm}$ GaAs nanowire exceeds 0.25 for photon energies higher than $1.5 \mathrm{eV}$. The maximum absorption efficiencies for nanowires in air (1.75) and on glass substrate (1.45) is observed at $2.51 \mathrm{eV}$. The case on the perfectly reflecting metal surface shows a peak absorption efficiency as high as 3.43 for a photon energy of $1.71 \mathrm{eV}$. For photon energies above $2.9 \mathrm{eV}$ the absorption efficiency saturates around $\sim 1$ for all three cases. This corresponds to the regime where the absorption length is significantly shorter than the diameter of the nanowire.

Now we turn our attention towards the total value of the EQE/IQE ratio in typical solar illumination conditions. We sum the weighted EQE/IQE ratio for energies between band gap $(1.42 \mathrm{eV})$ and $3.5 \mathrm{eV}$ with respect to the incoming photon flux of an AM1.5G solar spectrum. The resulting value corresponds to the fraction of photons with energy above the band-gap that actually generates an electron-hole pair. In Figure 10 we show the AM1.5G weighted EQE/IQE values for nanowire diameters in the range of 10-300 nm. The figure shows the EQE/IQE ratio of GaAs nanowires for illumination with unpolarized light for the three considered substrate conditions.

For nanowire diameters above $150 \mathrm{~nm}$ the EQE/IQE for GaAs nanowires on glass under AM1.5G spectral illumination is only in the oder of 0.5. This means that only about every second photon with energy above the band-gap is able to create an electron-hole pair in the nanowire. At the same time the ideally reflecting metal 


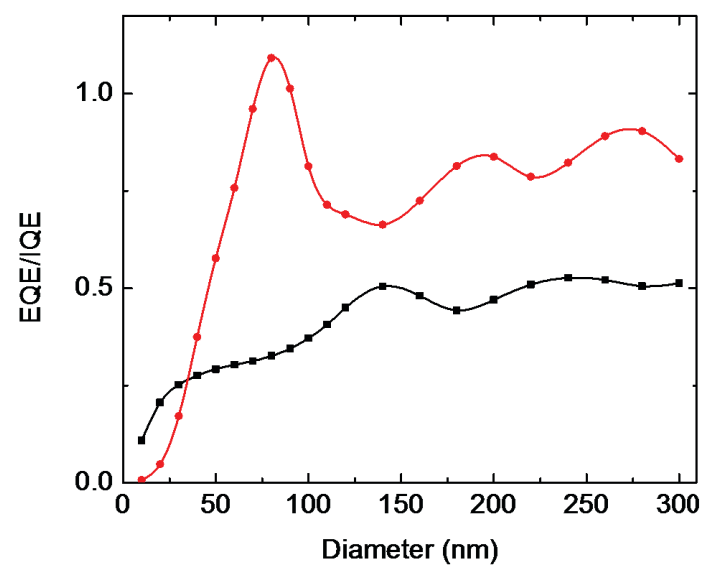

Figure 10. Photons absorbed in the nanowire normalized to the geometrically incident flux of photons of an AM1.5G solar spectrum on the projected surface area. Only photons with an energy above the band-gap are considered. The data is shown in dependence of wire diameter for cases of nanowire on glass substrate ( $\mathbf{\square})$ and on an ideally reflecting metal surface $(\bullet)$.

substrate $(\epsilon=-\infty)$ shows an improvement of light absorption by a factor of $\sim 1.5$ with respect to the case without any substrate. For the GaAs nanowire on the ideally reflecting substrate under illumination with an AM1.5G spectra, the calculation yield a peak EQE/IQE of 1.1. For this condition the nanowire will absorb more photons that what are geometrically incident to its projected surface area.

In typical experiments with single nanowire $\mathrm{p}(\mathrm{i}) \mathrm{n}$ junctions the conversion efficiency is calculated by normalizing the obtained solar power of the device to the incident solar power on the geometrically projected area. We have shown that for almost the entire range of experimental conditions the ratio EQE/IQE, and therefore the "effective" projected collection area is quite smaller than unity. As a consequence, the IQE could significantly deviate from the measured EQE.

In conclusion we have shown the influence of the configuration of single nanowire solar cells in the measurement of the conversion efficiency. The geometry used for nanowires mounted planar on a substrate can lead to the systematic measurement of lower values, which are a consequence of the reduced coupling of the incident light in the nanowire. The calculated EQE/IQE ratio for GaAs nanowires illuminated by a AM1.5G spectrum can be used as a reference for converting experimental efficiency values into the internal efficiency of the devices. This will enable a better comparison between the groups and materials systems.

\section{ACKNOWLEDGMENTS}

$\mathrm{MH}$ and AFiM acknowledge the support from G. Abstreiter and the projects MEXT SENFED, and Nanosystems Intitiative Munich in some of the research reviewed here. The authors thank financial support of by the Swiss National Science Foundation under Grant No. 2000021-121758/1 and 129775/1 by the European Research Council under Grant "Upcon".

\section{REFERENCES}

[1] Tsakalakos, L., "Nanostructures for photovoltaics," Materials Science and Engineering: R: Reports 62(6), 175 - 189 (2008).

[2] Tian, B., Kempa, T. J., and Lieber, C. M., "Single nanowire photovoltaics," Chemical Society Reviews 38(1), 16-24 (2009).

[3] Kayes, B. M., Atwater, H. A., and Lewis, N. S., "Comparison of the device physics principles of planar and radial p-n junction nanorod solar cells," Journal of Applied Physics 97(11), 114302 (2005). 
[4] Hu, L. and Chen, G., "Analysis of Optical Absorption in Silicon Nanowire Arrays for Photovoltaic Applications," Nano Letters 7(11), 3249-3252 (2007).

[5] Glas, F., "Critical dimensions for the plastic relaxation of strained axial heterostructures in free-standing nanowires," Phys. Rev. B 74, 121302 (Sep 2006).

[6] Kandala, A., Betti, T., and i Morral, A. F., "General theoretical considerations on nanowire solar cell designs," physica status solidi (a) 206(1), 173-178 (2009).

[7] Uccelli, E., Arbiol, J., Magen, C., Krogstrup, P., Russo-Averchi, E., Heiss, M., Mugny, G., Morier-Genoud, F., Nygard, J., Morante, J. R., and Fontcuberta i Morral, A., "Three-Dimensional Multiple-Order Twinning of Self-Catalyzed GaAs Nanowires on Si Substrates," Nano Letters (2011).

[8] Fontcuberta i Morral, A., Spirkoska, D., Arbiol, J., Heigoldt, M., Morante, J. R., and Abstreiter, G., "Prismatic Quantum Heterostructures Synthesized on Molecular-Beam Epitaxy GaAs Nanowires," Small 4(7), 899-903 (2008).

[9] Fontcuberta i Morral, A., Colombo, C., Abstreiter, G., Arbiol, J., and Morante, J. R., "Nucleation mechanism of gallium-assisted molecular beam epitaxy growth of gallium arsenide nanowires," Appl. Phys. Lett. 92(6), 063112 (2008).

[10] Fontcuberta i Morral, A., "Gold-Free GaAs Nanowire Synthesis and Optical Properties," Selected Topics in Quantum Electronics, IEEE Journal of 17, 819 -828 (july-aug. 2011).

[11] Henry, C. H., "Limiting efficiencies of ideal single and multiple energy gap terrestrial solar cells," Journal of Applied Physics 51(8), 4494-4500 (1980).

[12] Colombo, C., Spirkoska, D., Frimmer, M., Abstreiter, G., and Fontcuberta i Morral, A., "Ga-assisted catalyst-free growth mechanism of GaAs nanowires by molecular beam epitaxy," Phys. Rev. B 77(15), 155326 (2008).

[13] Heigoldt, M., Arbiol, J., Spirkoska, D., Rebled, J. M., Conesa-Boj, S., Abstreiter, G., Peiro, F., Morante, J. R., and i Morral, A. F., "Long range epitaxial growth of prismatic heterostructures on the facets of catalyst-free GaAs nanowires," Journal of Materials Chemistry 19(7), 840-848 (2009).

[14] Uccelli, E., Arbiol, J., Morante, J. R., and Fontcuberta i Morral, A., "InAs Quantum Dot Arrays Decorating the Facets of GaAs Nanowires," ACS Nano 4(10), 5985-5993 (2010).

[15] Demichel, O., Heiss, M., Bleuse, J., Mariette, H., and i Morral, A. F., "Impact of surfaces on the optical properties of GaAs nanowires," Appl. Phys. Lett. 97(20), 201907 (2010).

[16] Sze, S. M., [Semiconductor Devices: Physics and Technology], John Wiley \& Sons, 2nd edition ed. (2001).

[17] Suzuki, T. and Ogawa, M., "Degradation of photoluminescence intensity caused by excitation-enhanced oxidation of GaAs surfaces," Appl. Phys. Lett. 31(7), 473-475 (1977).

[18] Guidotti, D., Hasan, E., Hovel, H., and Albert, M., "Model for degradation of band gap photo-luminescence in GaAs," Il Nuovo Cimento D 11, 583-613 (Apr. 1989).

[19] Wittry, D. B. and Kyser, D. F., "Measurement of Diffusion Lengths in Direct-Gap Semiconductors by Electron-Beam Excitation," Journal of Applied Physics 38(1), 375-382 (1967).

[20] Sturge, M. D., "Optical Absorption of Gallium Arsenide between 0.6 and 2.75 eV," Phys. Rev. 127, 768-773 (Aug 1962).

[21] Williams, E. W. and Chapman, R. A., "Temperature Dependence of Photoluminescence in Cadmium-Doped Epitaxial GaAs," Journal of Applied Physics 38(6), 2547-2552 (1967).

[22] Rosenwaks, Y., Shapira, Y., and Huppert, D., "Picosecond time-resolved luminescence studies of surface and bulk recombination processes in InP," Phys. Rev. B 45, 9108-9119 (Apr 1992).

[23] Ando, K., Yamamoto, A., and Yamaguchi, M., "Surface Band Bending Effects on Photoluminescence Intensity in n-InP Schottky and MIS Diodes," Japanese Journal of Applied Physics 20(6), 1107-1112 (1981).

[24] Stroscio, J. A., Feenstra, R. M., and Fein, A. P., "Local state density and long-range screening of adsorbed oxygen atoms on the GaAs(110) surface," Phys. Rev. Lett. 58, 1668-1671 (Apr 1987).

[25] Ishikawa, Y., Tsurumi, N., Fukui, T., and Hasegawa, H., "Scanning tunneling microscopy and x-ray photoelectron spectroscopy studies of atomic level structure and Fermi level pinning on GaAs(110) surfaces grown by molecular beam epitaxy," Papers from the 25th annual conference on the physics and chemistry of semiconductor interfaces 16(4), 2387-2394, AVS (1998). 
[26] Bartels, F., Surkamp, L., Clemens, H. J., and Mönch, W., "Oxygen and hydrogen adsorption on GaAs(110)," Journal of Vacuum Science \& Technology B: Microelectronics and Nanometer Structures 1(3), 756-762 (1983).

[27] Su, C. Y., Lindau, I., Chye, P. W., Skeath, P. R., and Spicer, W. E., "Photoemission studies of the interaction of oxygen with GaAs(110)," Phys. Rev. B 25, 4045-4068 (Mar 1982).

[28] Landgren, G., Ludeke, R., Jugnet, Y., Morar, J. F., and Himpsel, F. J., "The oxidation of GaAs(110): A reevaluation," Journal of Vacuum Science 83 Technology B: Microelectronics and Nanometer Structures 2(3), 351-358 (1984).

[29] Fukasawa, R., Wakaki, M., Ohta, K., and Okumura, H., "Raman Scattering Determination of Free Carrier Concentration and Surface Depletion Layer in (100) p-GaAs Grown by Molecular-Beam Epitaxy," Japanese Journal of Applied Physics 25(Part 1, No. 4), 652-653 (1986).

[30] Sakamoto, N., Hirakawa, K., and Ikoma, T., "Conduction-type conversion in Si-doped (311)A GaAs grown by molecular beam epitaxy," Appl. Phys. Lett. 67(10), 1444-1446 (1995).

[31] Gardner, N. R., Woods, N. J., Domnguez, P. S., Tok, E. S., Norman, C. E., and Harris, J. J., "Electrical properties of lateral p - n junctions formed on patterned (110) GaAs substrates," Semiconductor Science and Technology 12(6), 737 (1997).

[32] Dufouleur, J., Colombo, C., Garma, T., Ketterer, B., Uccelli, E., Nicotra, M., and Fontcuberta i Morral, A., "P-Doping Mechanisms in Catalyst-Free Gallium Arsenide Nanowires," Nano Letters 10(5), 1734-1740 (2010).

[33] Colombo, C., Heiß, M., Gratzel, M., and Fontcuberta i Morral, A., "Gallium arsenide p-i-n radial structures for photovoltaic applications," Appl. Phys. Lett. 94(17), 173108 (2009).

[34] Tian, B., Zheng, X., Kempa, T. J., Fang, Y., Yu, N., Yu, G., Huang, J., and Lieber, C. M., "Coaxial silicon nanowires as solar cells and nanoelectronic power sources," Nature 449, 885-889 (Oct. 2007).

[35] Kelzenberg, M. D., Turner-Evans, D. B., Kayes, B. M., Filler, M. A., Putnam, M. C., Lewis, N. S., and Atwater, H. A., "Photovoltaic Measurements in Single-Nanowire Silicon Solar Cells," Nano Letters 8(2), 710-714 (2008). PMID: 18269257.

[36] Kempa, T. J., Tian, B., Kim, D. R., Hu, J., Zheng, X., and Lieber, C. M., "Single and Tandem Axial p-i-n Nanowire Photovoltaic Devices," Nano Letters 8(10), 3456-3460 (2008). PMID: 18763836.

[37] Czaban, J. A., Thompson, D. A., and LaPierre, R. R., "GaAs Core-Shell Nanowires for Photovoltaic Applications," Nano Letters 9(1), 148-154 (2009).

[38] Aspnes, D. E. and Studna, A. A., "Dielectric functions and optical parameters of Si, Ge, GaP, GaAs, GaSb, InP, InAs, and InSb from 1.5 to 6.0," Phys. Rev. B 27, 985 (Jan 1983).

[39] Fan, Z., Ruebusch, D., Rathore, A., Kapadia, R., Ergen, O., Leu, P., and Javey, A., "Challenges and prospects of nanopillar-based solar cells," Nano Research 2, 829-843 (2009).

[40] Maiolo, J., Kayes, B., Filler, M., Putnam, M., Kelzenberg, M., Atwater, H., and Lewis, N., "High Aspect Ratio Silicon Wire Array Photoelectrochemical Cells," J. Am. Chem. Soc. 129(41), 12346-12347 (2007).

[41] Garnett, E. and Yang, P., "Light Trapping in Silicon Nanowire Solar Cells," Nano Letters 10(3), 1082-1087 (2010).

[42] Ruda, H. E. and Shik, A., "Polarization-sensitive optical phenomena in thick semiconducting nanowires," Journal of Applied Physics 100(2), 024314 (2006).

[43] Cao, L., White, J. S., Park, J.-S., Schuller, J. A., Clemens, B. M., and Brongersma, M. L., "Engineering light absorption in semiconductor nanowire devices," Nat Mater 8, 643-647 (Aug. 2009).

[44] Goto, H., Nosaki, K., Tomioka, K., Hara, S., Hiruma, K., Motohisa, J., and Fukui, T., "Growth of CoreShell InP Nanowires for Photovoltaic Application by Selective-Area Metal Organic Vapor Phase Epitaxy," Applied Physics Express 2(3), 035004 (2009). 